\title{
Development of Belle-II TOP detector and its MCP-PMT
}

\author{
Kenji Inami ${ }^{* \dagger}$ \\ Nagoya Univ. \\ E-mail: kenji@hepl.phys.nagoya-u.ac.jp
}

We have developed a Cherenkov ring-imaging detector as a barrel particle identification device, named TOP, for the Belle II experiment. We have successfully produced the detector modules, which consists of the $2.7 \mathrm{~m}$ long quartz radiators, MCP-PMTs and readout electronics, by developing many tools and method. Along with the construction, we also have developed the lifeextended MCP-PMT with the Hamamatsu photonics, to keep the performance for long period even under higher background environment than the expected rate at SuperKEKB.

38th International Conference on High Energy Physics

3-10 August 2016

Chicago, USA

* Speaker.

${ }^{\dagger}$ Belle II PID group 


\section{Introduction}

We have been developed new ring imaging Cherenkov detector, named "TOP detector" [1], for the $\pi^{ \pm} / K^{ \pm}$particle identification (PID) in the barrel region of Belle II detector [2], which improves the PID performance. The target fake rate is about 5 times less than the previous Belle experiment.

TOP detector measures the time-of-flight and time-of-propagation of internally reflected Cherenkov photons, emitted by the charged particle. TOP consists of the large quartz radiators, a focusing mirror and an image expansion block, as shown in Fig. 1(left). The mirror and expansion block is used to correct the chromaticity and increase the number of effective detected photons. The quartz radiator is made of high quality fused-silica with a polishing accuracy of $2 \lambda$ flatness and $5 \AA$ surface roughness. Two quartz bars, a focusing mirror and an expansion block are glued together (Fig. 1(right)) with an angle accuracy of $\sim 0.02 \mathrm{mrad}$ by EPOTEK305-2 epoxy adhesive.
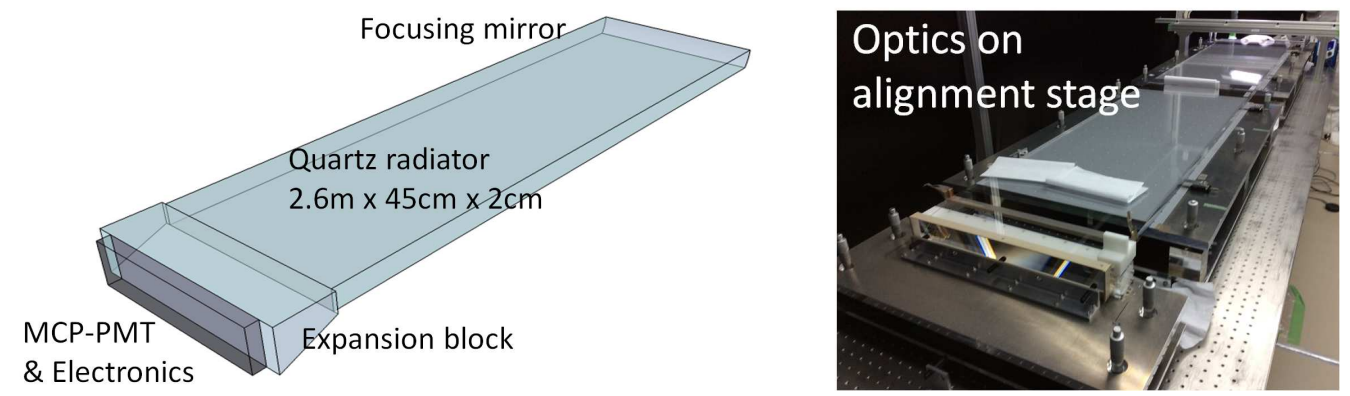

Figure 1: Overview of TOP radiator shape and readout position (left) and the photo of the quartz radiator on the alignment stage (right). TOP detector consists of the quartz radiator with the size of $2.6 \mathrm{~m} \times 45 \mathrm{~cm}$ $\times 2 \mathrm{~cm}$ and focusing mirror and expansion block. At the end of the radiator, MCP-PMTs and readout electronics are attached.

The radiator is supported by PEEK buttons on the honeycomb panel. The button surface are tuned within $\sim 40 \mu \mathrm{m}$ flatness. The module stiffness is improved by adopting round-shaped honeycomb panel, and also by connecting the modules each other as barrel shape. During the module production, we attached "strong back" support frame to the quartz-bar-box (QBB), to keep the flatness within $500 \mu \mathrm{m}$.

On the end of expansion block, photon detectors and the readout electronics are attached. We have employed square-shape MCP-PMTs [3] as the photon detector, which can detect single photon even under a $1.5 \mathrm{~T}$ magnetic field, with the transit-time-spread of $<40 \mathrm{ps}$ (Fig. 2). The PMT signal is read out by a newly developed ASIC [4] with GHz analog bandwidth, high performance waveform recording, to achieve pico-second level timing measurement.

\section{MCP-PMT}

We have developed the MCP-PMT with Hamamatsu photonics K.K., to obtain the higher performance, especially for the single photon detection efficiency. Along with the mass production for Belle II experiment, we have tuned the photocathode recipe and obtained the high quantum efficiency (QE) of $28 \sim 31 \%$ at the peak wavelength. The typical QE distribution is shown in Fig. 3(left). 

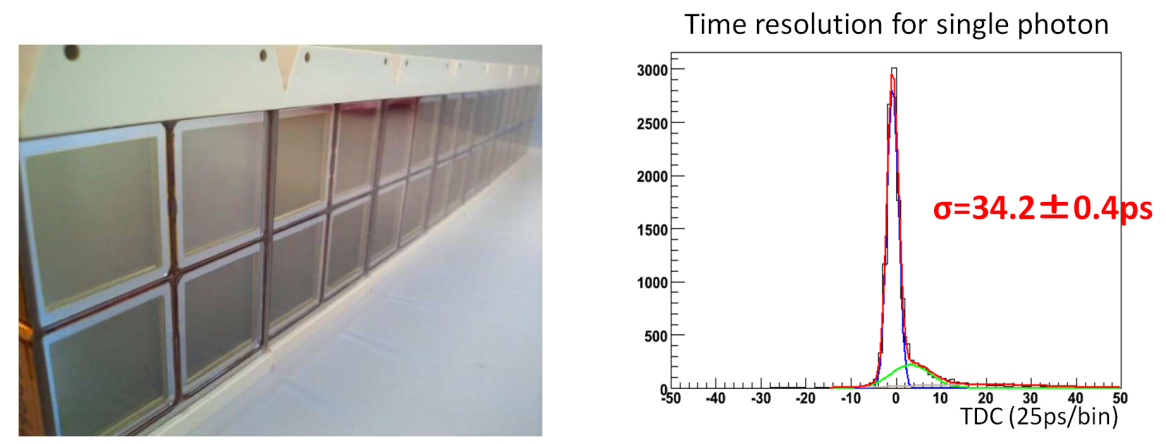

Figure 2: Photo of MCP-PMT array for TOP detector (left) and typical timing distribution for single photon detection by pulse laser (right).

The photocathode lifetime was main issue for high intensity experiments. In the Belle II, many background gammas from the beam-line illuminate the quartz radiator and covert to electronpositron pairs, which emit background Cherenkov photons and makes many background signals at MCP-PMT. The photocathode is damaged by feed-backed ions from MCP due to the electron multiplication process. In order to improve the lifetime, we adopted ALD coated MCP [5] and also developed further life-extended MCP-PMT with Hamamatsu photonics by tuning the MCP cleaning method. The result is shown in Fig. 3(right).
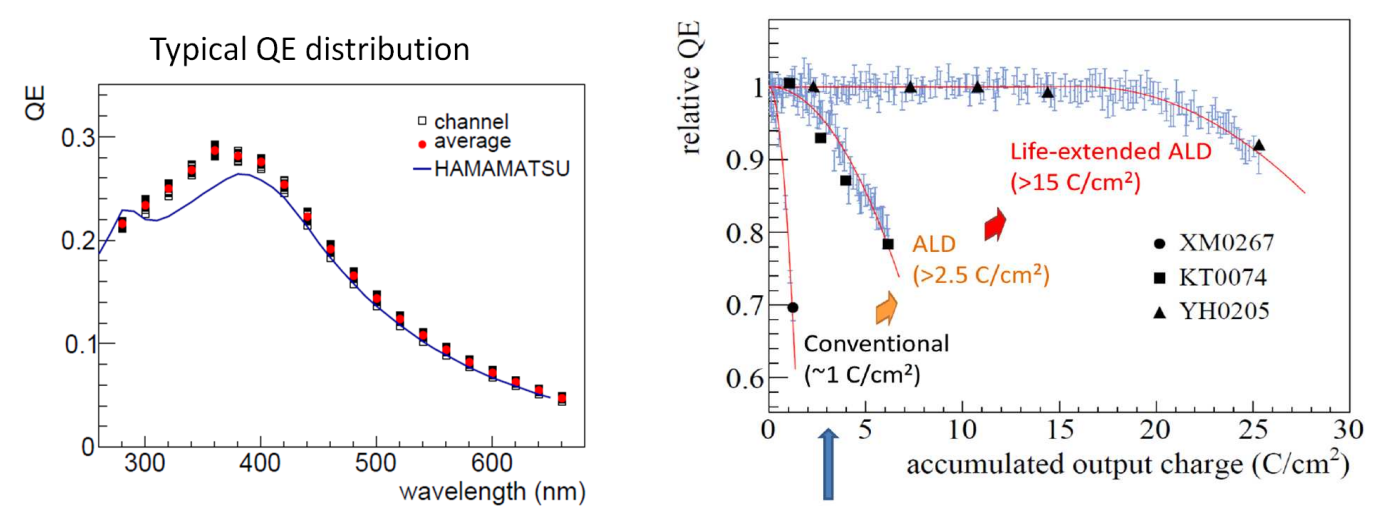

Figure 3: Typical QE distribution as a function of photon wavelength (left) and the relative QE depending on the output charge (right).

\section{TOP module production}

Finally, we have started the TOP module production from December 2014, following the practice of 4 prototypes. After optics gluing and honeycomb/button preparation, we lift up the optics from the gluing stage to honeycomb panel (Fig. 4(left)), then assemble the QBB, PMT modules and front-end electronics. We have produced the TOP modules with 2.5 weeks per module, successfully, and finished in about one year. Then we have installed into Belle II structure in April/May 2016 (Fig. 4(right)). The test with cosmic-ray is underway. 

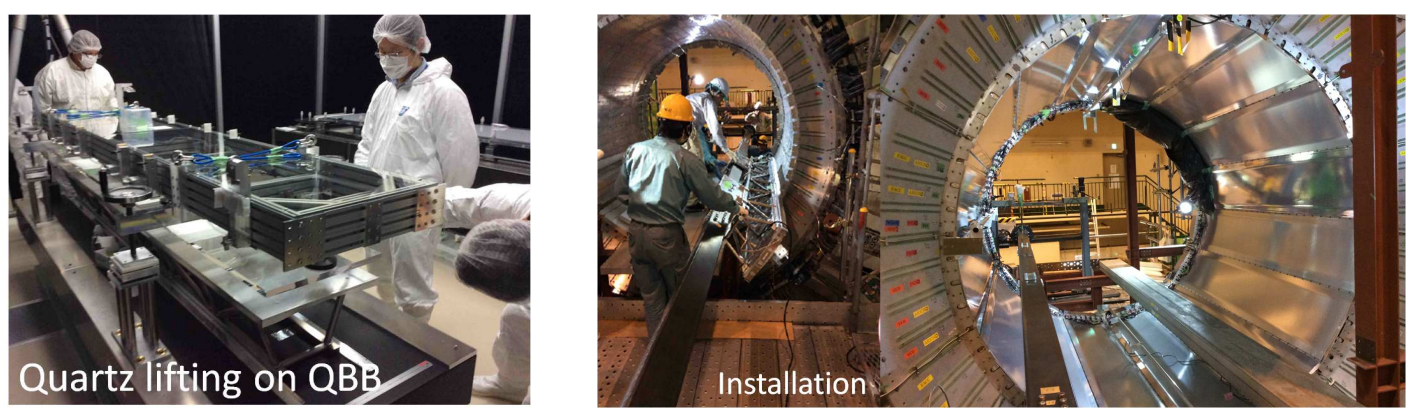

Figure 4: Photos of TOP module production (left) and the installation (right).

\section{References}

[1] M. Akatsu et al., Nucl. Instr. and Meth. A 440 (2000) 124-135; T. Ohshima, Nucl. Instr. and Meth. A453 (2000) 331-335; M. Hirose et al., Nucl. Instr. and Meth. A 460 (2001) 326-335; S. Matsui et al., Nucl. Instr. and Meth. A 463 (2001) 220-226; Y. Enari et al., Nucl. Instr. and Meth. A 494 (2002) 430-435; T. Hokuue et al., Nucl. Instr. and Meth. A 494 (2002) 436-440; Y. Enari et al., Nucl. Instr. and Meth. A 547 (2005) 490-503; K. Inami et al., Nucl. Instr. and Meth. A 560 (2006) 303-308.

[2] T. Abe et al. [Belle-II Collaboration], arXiv:1011.0352 [physics.ins-det].

[3] M. Akatsu et al., Nucl. Instr. and Meth. A 528 (2004) 763; K. Matsuoka, Nucl. Instr. and Meth. A 766 (2014) 148-151.

[4] M. Andrew, PoS TIPP2014 (2014) 171.

[5] O. Siegmund et al., Nucl. Instr. and Meth. A 695 (2012) 168. 